PROCEEDINGS OF THE

AMERICAN MATHEMATICAL SOCIETY

Volume 138, Number 8, August 2010, Pages 2839-2852

S 0002-9939(10)10345-1

Article electronically published on April 2, 2010

\title{
THE MODULATION MAPPING FOR MAGNETIC SYMBOLS AND OPERATORS
}

\author{
MARIUS MĂNTOIU AND RADU PURICE
}

(Communicated by Marius Junge)

\begin{abstract}
We extend the Bargmann transform to the magnetic pseudodifferential calculus, using gauge-covariant families of coherent states. We also introduce modulation mappings, a first step towards adapting modulation spaces to the magnetic case.
\end{abstract}

\section{INTRODUCTION}

Recent publications [19, 24, 25, 27, 17, 22 introduced and developed a mathematical formalism for the quantization of physical systems with variable magnetic fields. We would like now to complete the picture, sketching the connection with coherent states, the Bargmann transform and a suitable version of the modulation mapping.

Classically, the magnetic field changes the geometry of the phase space. This is realized by a modification of the standard symplectic form and, consequently, of the Poisson algebra structure of the smooth functions on phase space, interpreted as classical observables. Correspondingly, at the quantum level, one introduces [19, 24, 26] algebras of observables defined only in terms of the magnetic field, no choice of a vector potential being needed. The main new object is a composition law on symbols defined by fluxes of the magnetic field through triangles.

To get self-adjoint operators and a Hilbert space theory, the resulting algebras are represented in Hilbert spaces; this is done by choosing vector potentials defining the magnetic field. One thus gets essentially a new pseudodifferential calculus ([24, [17, 18), seen as a functional calculus for the family of non-commuting operators composed of positions and magnetic moments. When no magnetic field is present, it coincides with the Weyl quantization. One of its main virtues is gauge-covariance: equivalent choices of vector potentials lead to unitarily equivalent representations. We stress that this property is not shared by doing a minimal coupling modification of the symbol in the usual Weyl calculus.

Received by the editors July 30, 2009 and, in revised form, December 2, 2009.

2010 Mathematics Subject Classification. Primary 35S05, 47L15; Secondary 47L65, 47L90.

Key words and phrases. Magnetic field, pseudodifferential operator, phase space, modulation mapping, crossed product algebra, coherent states, Bargmann transform.

The first author is partially supported by Núcleo Cientifico ICM P07-027-F "Mathematical Theory of Quantum and Classical Magnetic Systems" and by the Chilean Science Foundation Fondecyt under grant no. 1085162. His interest in modulation spaces was raised by a very enjoyable visit to the University of Vienna in February 2009.

The second author acknowledges partial support from contract no. 2-CEx 06-11-18/2006.

(C)2010 American Mathematical Society Reverts to public domain 28 years from publication 2839 
Both the intrinsic and the represented version admit $C^{*}$-algebraic reformulations $([27,24])$. They were useful in the spectral analysis of magnetic Schrödinger operators; cf. [28, 23] for instance.

One of the purposes of this article is to define and study a modulation mapping in the setting of the magnetic quantum formalism. The main application of this magnetic modulation mapping would be inducing useful new function spaces on $\Xi$ from known function spaces on $\Xi \times \Xi$. This will be done in a future publication.

Modulation spaces are Banach function spaces introduced a long time ago by H. Feichtinger [5, 6]. By definition, they involve norm estimates on a certain family of transformations of the function one studies, defined on $\mathbb{R}^{n}$ or on a locally compact abelian group. Modulation spaces evolved especially in connection with Time Frequency Analysis, Gabor Frames and Signal Processing Theory. In [30, J. Sjöstrand discovered the importance of one of these spaces in the theory of pseudodifferential operators; cf. also [31]. Then the interconnection between modulation spaces and pseudodifferential theory developed considerably, as a result both of "the Vienna school" and other researchers. We cite, without any claim of completeness, 3, 4, 17, 9, 10, 11, 12, 14, 16, 32]. Other important works are cited in these articles.

To define modulation spaces, one introduces first a transformation (the Short Time Fourier Transform) from functions defined on the phase space $\Xi=\mathbb{R}^{2 N}$ to functions defined on $\Xi \times \Xi$; we are going to indicate in Section 2 a magnetic analog of this transformation. Since we are mainly interested in its behavior with respect to symbol composition, we deviate to a certain extent from the standard approach. Therefore, if $B=0$, one does not recover the usual expression leading to coorbit spaces, so our definition could have some interest even in the non-magnetic case. We show that this transformation is isometric between $L^{2}$-spaces and it transforms the magnetic analog of the Weyl composition law into the multiplication in a typical crossed product algebra, which can also be seen as the Kohn-Nirenberg composition for symbols defined in $\Xi \times \Xi$.

In Section 3 we show that our modulation mapping is an intrinsic counterpart of the transformation sending operators from the Schrödinger representation to the magnetic Bargmann representation. This one is induced by a proper choice of a family of coherent states; for the standard case as well as for many generalizations, we refer to [1, 8, 15, 22] and to the references therein. Our result says that magnetic Weyl operators can be seen as representations of a crossed product algebra or as usual Kohn-Nirenberg operators defined in $\mathbb{R}^{2 N}$. The symbols of these KohnNirenberg operators are computed from the magnetic Weyl symbol by applying the magnetic modulation mapping followed by a partial Fourier transform.

\section{Preliminaries on the magnetic Weyl Calculus}

In this section we recall the structure of the observable algebras of a particle in a variable magnetic field. We follow the references [24, [26] and [17, which contain further details and technical developments.

The physical system we consider consists in a spinless particle moving in the Euclidean space $\mathcal{X}:=\mathbb{R}^{N}$ under the influence of a magnetic field. We denote by $\mathcal{X}^{*}$ the dual space of $\mathcal{X}$. The duality is given simply by $\mathcal{X} \times \mathcal{X}^{*} \ni(x, \xi) \mapsto x \cdot \xi$. The phase space $\Xi:=T^{*} \mathcal{X} \equiv \mathcal{X} \times \mathcal{X}^{*}$, containing points $X=(x, \xi), Y=(y, \eta)$, $Z=(z, \zeta)$, is endowed with the standard symplectic form

$$
\sigma(X, Y) \equiv \sigma[(x, \xi),(y, \eta)]:=y \cdot \xi-x \cdot \eta .
$$


The magnetic field is a continuous closed 2-form $B$ on $\mathcal{X}(d B=0)$, given by matrix-component functions $B_{j k}=-B_{k j}: \mathcal{X} \rightarrow \mathbb{R}, j, k=1, \ldots, N$. It defines quantum observable composition in terms of its fluxes through triangles. If $a, b, c \in$ $\mathcal{X}$, then we denote by $\langle a, b, c\rangle$ the triangle in $\mathcal{X}$ of vertices $a, b$ and $c$ and set

$$
\Gamma^{B}(\langle a, b, c\rangle):=\int_{\langle a, b, c\rangle} B
$$

for the flux of $B$ through it (integration of a 2 -form on a 2 -simplex). Then the formula

$$
\begin{aligned}
& \left(f \#^{B} g\right)(X):=\pi^{-2 N} \int_{\Xi} d Y \int_{\Xi} d Z \exp [-2 i \sigma(X-Y, X-Z)] \\
& \quad \times \exp \left[-i \Gamma^{B}(\langle x-y+z, y-z+x, z-x+y\rangle)\right] f(Y) g(Z)
\end{aligned}
$$

defines a formal associative composition law on functions $f, g: \Xi \rightarrow \mathbb{C}$.

The formula (1.1) makes sense and has nice properties under various circumstances. For example, if the components $B_{j k}$ belong to $C_{\mathrm{pol}}^{\infty}(\mathcal{X})$, the class of smooth functions on $\mathcal{X}$ with polynomial bounds on all the derivatives, then the Schwartz space $\mathcal{S}(\Xi)$ is stable under $\#^{B}$. The dual of $\mathcal{S}(\Xi)$ being denoted by $\mathcal{S}^{*}(\Xi)$ (tempered distributions), one also has

$$
\#^{B}: \mathcal{S}(\Xi) \times \mathcal{S}^{*}(\Xi) \rightarrow \mathcal{S}^{*}(\Xi) \text { and } \#^{B}: \mathcal{S}^{*}(\Xi) \times \mathcal{S}(\Xi) \rightarrow \mathcal{S}^{*}(\Xi)
$$

Denoting by $\mathcal{M}^{B}(\Xi)$ the largest subspace of $\mathcal{S}^{*}(\Xi)$ for which

$$
\#^{B}: \mathcal{S}(\Xi) \times \mathcal{M}^{B}(\Xi) \rightarrow \mathcal{S}(\Xi) \text { and } \#^{B}: \mathcal{M}^{B}(\Xi) \times \mathcal{S}(\Xi) \rightarrow \mathcal{S}(\Xi),
$$

it can be shown that $\mathcal{M}^{B}(\Xi)$ is an involutive algebra under $\#^{B}$ and under complex conjugation, for which

$$
\#^{B}: \mathcal{S}^{*}(\Xi) \times \mathcal{M}^{B}(\Xi) \rightarrow \mathcal{S}^{*}(\Xi) \text { and } \#^{B}: \mathcal{M}^{B}(\Xi) \times \mathcal{S}^{*}(\Xi) \rightarrow \mathcal{S}^{*}(\Xi)
$$

This is quite a large class of distributions, containing all the bounded measures as well as the class $C_{\mathrm{pol}, \mathrm{u}}^{\infty}(\Xi)$ of all smooth functions for which all the derivatives are bounded by some polynomial (depending on the function, but not on the order of the derivative). In addition, if we assume that all the derivatives of the functions $B_{j k}$ are bounded, the Hörmander classes of symbols $S_{\rho, \delta}^{m}(\Xi)$ composes in the usual way under $\#^{B}$.

Being a closed 2-form in $\mathcal{X}=\mathbb{R}^{N}$, the magnetic field is exact: it can be written as $B=d A$ for some continuous 1-form $A$ (called the vector potential). Vector potentials enter by their circulations

$$
\Gamma^{A}([x, y]):=\int_{[x, y]} A
$$

through segments $[x, y]:=\{t x+(1-t) y \mid t \in[0,1]\}$. For a vector potential $A$ with $d A=B$, let us define

$$
\left[\mathfrak{O p}^{A}(f) u\right](x)
$$

$$
:=(2 \pi)^{-N} \int_{\mathcal{X}} \int_{\mathcal{X}^{*}} d y d \xi \exp [i(x-y) \cdot \xi] \exp \left[-i \Gamma^{A}([x, y])\right] f\left(\frac{x+y}{2}, \xi\right) u(y) .
$$

For $A=0$ one recognizes the Weyl quantization, associating to functions or distributions on $\Xi$ linear operators acting on function spaces on $\mathcal{X}$. 
The space $L^{2}(\Xi)$ is a ${ }^{*}$-algebra under $\#^{B}$ and complex conjugation and $\mathfrak{O} \mathfrak{p}^{A}$ is an isomorphism of $L^{2}(\Xi)$ on the Hilbert space $\mathbb{B}_{2}(\mathcal{H})$ of all the Hilbert-Schmidt operators on $\mathcal{H}=L^{2}(\mathcal{X})$.

Suitably interpreted (by using duality arguments), $\mathfrak{O p}^{A}$ defines a representation of the ${ }^{*}$-algebra $\mathcal{M}^{B}(\Xi)$ by linear continuous operators : $\mathcal{S}(\mathcal{X}) \rightarrow \mathcal{S}(\mathcal{X})$, i.e.

$$
\mathfrak{O p}^{A}\left(f \#^{B} g\right)=\mathfrak{O} \mathfrak{p}^{A}(f) \mathfrak{O p} \mathfrak{p}^{A}(g) \text { and } \mathfrak{O p}^{A}(\bar{f})=\mathfrak{O p}^{A}(f)^{*}
$$

for any $f, g \in \mathcal{M}^{B}(\Xi)$. In addition, $\mathfrak{O p}^{A}$ restricts to an isomorphism from $\mathcal{S}(\Xi)$ to $\mathbb{B}\left[\mathcal{S}^{*}(\mathcal{X}), \mathcal{S}(\mathcal{X})\right]$ and extends to an isomorphism from $\mathcal{S}^{*}(\Xi)$ to $\mathbb{B}\left[\mathcal{S}(\mathcal{X}), \mathcal{S}^{*}(\mathcal{X})\right]$ (we set $\mathbb{B}(\mathcal{R}, \mathcal{T})$ for the family of all linear continuous operators between the topological vector spaces $\mathcal{R}$ and $\mathcal{T}$ ).

An important property of (1.2) is gauge covariance: if $A^{\prime}=A+d \rho$ defines the same magnetic field as $A$, then $\mathfrak{O} p^{A^{\prime}}(f)=e^{i \rho} \mathfrak{O p}{ }^{A}(f) e^{-i \rho}$. Such a property would not hold for the wrong quantization, appearing in the literature as

$$
\begin{gathered}
{\left[\mathcal{O} p_{A}(f) u\right](x)} \\
:=(2 \pi)^{-N} \int_{\mathcal{X}} \int_{\mathcal{X}^{*}} d y d \xi \exp [i(x-y) \cdot \xi] f\left(\frac{x+y}{2}, \xi-A\left(\frac{x+y}{2}\right)\right) u(y) .
\end{gathered}
$$

To justify (1.2) we define a family $\left(\mathfrak{e}_{X}\right)_{X \in \Xi}$ of functions that will play an important role in the sequel:

$$
\mathfrak{e}_{X}(Z):=\exp \{-i \sigma(X, Z)\}, \quad Z \in \Xi .
$$

Actually they are elements of $C_{\mathrm{pol}, \mathrm{u}}^{\infty}(\Xi) \subset \mathcal{M}^{B}(\Xi)$. One checks easily that

$$
\mathfrak{e}_{X} \#^{B} \mathfrak{e}_{Y}=\Omega^{B}(X, Y) \#^{B} \mathfrak{e}_{X+Y},
$$

where $\Omega^{B}: \Xi \times \Xi \rightarrow C(\mathcal{X}, U(1))$ is the 2-cocycle defined by the canonical symplectic form and by the magnetic field $B$,

$$
\Omega^{B}(X, Y)(z) \equiv \Omega^{B}(X, Y ; z):=\exp \left[\frac{i}{2} \sigma(X, Y)\right] \omega^{B}(X, Y ; z),
$$

with

$$
\omega^{B}(X, Y ; z):=\exp \left[-i \Gamma^{B}(\langle z, z+x, z+x+y\rangle)\right] .
$$

Suitable functions $f: \Xi \rightarrow \mathbb{C}$ can be expressed as

$$
f(Y)=(2 \pi)^{-N} \int_{\Xi} d X(\mathfrak{F} f)(X) e^{-i \sigma(X, Y)}=(2 \pi)^{-N} \int_{\Xi} d X(\mathfrak{F} f)(X) \mathfrak{e}_{X}(Y),
$$

where $\mathfrak{F} f$ is the symplectic Fourier transform of $f$, so a good quantization should have the property

$$
\mathfrak{O p}^{A}(f)=(2 \pi)^{-N} \int_{\Xi} d X(\mathfrak{F} f)(X) \mathfrak{O p}^{A}\left(\mathfrak{e}_{X}\right) .
$$

Thus, the problem is to justify a choice for the operators $\mathfrak{o p}^{A}(X):=\mathfrak{O p}^{A}\left(\mathfrak{e}_{X}\right)$ acting in $\mathcal{H}=L^{2}(\mathcal{X})$.

In the presence of a magnetic field $B=d A$, a basic family of self-adjoint operators is $\left(Q_{1}, \ldots, Q_{N} ; \Pi_{1}^{A}, \ldots, \Pi_{N}^{A}\right)$, where $Q_{j}$ is the operator of multiplication by the coordinate function $x_{j}$ and $\Pi_{j}^{A}:=-i \partial_{j}-A_{j}$ is the $j$-th component of the magnetic moment. They satisfy the commutation relations

$$
i\left[Q_{j}, Q_{k}\right]=0, \quad i\left[\Pi_{j}^{A}, Q_{k}\right]=\delta_{j k}, \quad i\left[\Pi_{j}^{A}, \Pi_{j}^{A}\right]=B_{j k} .
$$


One gets (1.2) as a consequence of (1.6), admitting that the quantization of the function $X \mapsto \mathfrak{e}_{Y}(X)$ should be the unitary operator

$$
\begin{aligned}
\mathfrak{o p}^{A}(y, \eta) & :=\mathfrak{O p}^{A}\left(\mathfrak{e}_{(y, \eta)}\right) \\
& =\exp \left[-i \sigma\left((y, \eta),\left(Q, \Pi^{A}\right)\right)\right]=\exp \left[-i\left(Q \cdot \eta-y \cdot \Pi^{A}\right)\right],
\end{aligned}
$$

given by the explicit formula

$$
\left[\mathfrak{o p}^{A}(y, \eta) u\right](x)=e^{-i\left(x+\frac{y}{2}\right) \cdot \eta} e^{-i \Gamma^{A}([x, x+y])} u(x+y), \quad x, y \in \mathcal{X}, \eta \in \mathcal{X}^{*}, u \in \mathcal{H} .
$$

As a consequence of (1.3), one has

$$
\mathfrak{o p}^{A}(X) \mathfrak{o p}^{A}(Y)=\Omega^{B}(X, Y ; Q) \mathfrak{o p}^{A}(X+Y), \quad \forall X, Y \in \Xi,
$$

where $\Omega^{B}(X, Y ; Q)$ is the operator of multiplication by the function

$$
z \mapsto\left[\Omega^{B}(X, Y)\right](z) \equiv \Omega^{B}(X, Y ; z)
$$

given at (1.4) and (1.5).

The operator norm $\|\cdot\|$ on $\mathbb{B}(\mathcal{H})$ being relevant to Quantum Mechanics, we pull it back to symbols (the nicer, completely intrinsic approach can be found in [27], [26]). So let us set

$$
\|\cdot\|^{B}: \mathcal{S}(\Xi) \rightarrow \mathbb{R}_{+}, \quad\|f\|^{B}=\left\|\mathfrak{O p p}^{A}(f)\right\| .
$$

By gauge covariance, it is clear that $\|\cdot\|^{B}$ depends only on the magnetic field $B$ and not on the vector potential $A$. We denote by $\mathfrak{A}^{B}(\Xi)$ the completion of $\mathcal{S}(\Xi)$ under $\|\cdot\|^{B}$. It is a $C^{*}$-algebra that can be identified to a vector subspace of $\mathcal{S}^{*}(\Xi)$ and $\mathfrak{O p}^{A}: \mathfrak{A}^{B}(\Xi) \rightarrow \mathbb{B}(\mathcal{H})$ is a faithful *-representation, with $\mathfrak{O p}^{A}\left[\mathfrak{A}^{B}(\Xi)\right]=$ $\mathbb{K}(\mathcal{H})$, the $C^{*}$-algebra of compact operators in $\mathcal{H}$.

Many other useful $C^{*}$-algebras can be defined in this manner. An important one is $\mathfrak{C}^{B}(\Xi)$, defined such that $\mathfrak{O p}^{A}: \mathfrak{C}^{B}(\Xi) \rightarrow \mathbb{B}(\mathcal{H})$ is an isomorphism. The "magnetic version" of the Calderon-Vaillancourt theorem, proved in [17, says that if $B_{j k} \in B C^{\infty}(\mathcal{X}), \quad j, k=1, \ldots, N$, then the Fréchet space $B C^{\infty}(\Xi)$ of smooth functions on $\Xi$ having bounded derivatives of any order is continuously embedded in $\mathfrak{C}^{B}(\Xi)$. We note that $L^{2}(\Xi)$ and $\mathfrak{A}^{B}(\Xi)$ are ${ }^{*}$-ideals in $\mathfrak{C}^{B}(\Xi)$.

We set

$$
\Theta_{Z}^{B}(f):=\mathfrak{e}_{-Z} \#^{B} f \#^{B} \mathfrak{e}_{Z}
$$

for the family of magnetic translations in phase space, introduced in [18, and used for a Beals-type characterization of magnetic pseudodifferential operators by commutators. They are automorphisms of the ${ }^{*}$-algebras $L^{2}(\Xi), \mathfrak{A}^{B}(\Xi), \mathfrak{C}^{B}(\Xi), \mathfrak{M}^{B}(\Xi)$ and reduce, for $B=0$, to the usual translations $\left[\Theta_{Z}(f)\right](X):=f(X+Z)$.

We shall need an explicit form of $\Theta_{Z}^{B}$, obtained in [18. For this we define the following commutative mixed product (a mixture between point-wise multiplication in the first variable and convolution in the second)

$$
(F \star g)(x, \xi):=\int_{\mathcal{X}^{\prime}} d \eta F(x, \xi-\eta) g(x, \eta) .
$$

Proposition 1.1. For any points $x, y, z \in \mathcal{X}$ let us define the parallelogram

$$
\mathcal{P}(x ; y, z):=\{x+s y+t z \mid s \in[-1 / 2,1 / 2], t \in[-1,0]\},
$$


having edges parallel to the vectors $y$ and $z$, respectively. We consider the distribution

$$
\begin{gathered}
\Omega^{B}[\mathcal{P}(x ; y, z)]=\exp \left\{-i \Gamma^{B}[\mathcal{P}(x ; y, z)]\right\} \\
=\exp \left\{-i \sum_{j, k=1}^{N} y_{j} z_{k} \int_{-1 / 2}^{1 / 2} d s \int_{-1}^{0} d t B_{j k}(x+s y+t z)\right\}
\end{gathered}
$$

and its Fourier transform with respect to the second variable:

$$
\widetilde{\Omega_{\mathcal{P}}^{B}}[z](x, \xi):=(2 \pi)^{-N} \int_{\mathcal{X}} d y e^{-i y \cdot \xi} \Omega^{B}[\mathcal{P}(x ; y, z)] .
$$

For $Z=(z, \zeta) \in \Xi$ and $f \in L^{1}(\Xi)$ we have

$$
\Theta_{Z}^{B}(f)=\widetilde{\Omega_{\mathcal{P}}^{B}}[z] \star \Theta_{Z}[f] .
$$

More generally, we set

$$
\Theta_{Y, Z}^{B}(f):=\mathfrak{e}_{-Y} \#^{B} f \#^{B} \mathfrak{e}_{Y-Z}
$$

which makes sense for every $f \in \mathcal{M}^{B}(\Xi)$. We have $\Theta_{Y, 0}^{B}=\Theta_{Y}^{B}$.

\section{Modulation mappings}

We only assume that $B$ has continuous components. On functions defined on $\Xi$ or on $\Xi \times \Xi$, respectively, we will use the "real" scalar products

$$
\langle f, g\rangle:=\int_{\Xi} d X f(X) g(X), \quad\langle\langle F, G\rangle\rangle:=\int_{\Xi} \int_{\Xi} d X d Y F(X, Y) G(X, Y) .
$$

Definition 2.1. The magnetic modulation mapping $M_{h}^{B}: L^{2}(\Xi) \rightarrow L^{2}(\Xi \times \Xi)$ associated to $h \in L^{2}(\Xi)$ is

$$
\left[M_{h}^{B}(f)\right](X, Y):=\left\langle\mathfrak{e}_{-X} \#^{B} f \#^{B} \mathfrak{e}_{X-Y}, h\right\rangle=\left\langle\Theta_{X, Y}^{B}[f], h\right\rangle .
$$

Remark. By using the formula (2.1), one can also write

$$
\left[M_{h}^{B}(f)\right](X, Y)=\left\langle h \#^{B} \mathfrak{e}_{-X} \#^{B} f, \mathfrak{e}_{X-Y}\right\rangle=\left[\mathfrak{F}\left(h \#^{B} \mathfrak{e}_{-X} \#^{B} f\right)\right](X-Y) .
$$

In the case $B=0$ one gets

$$
\left[M_{h}(f)\right](X, Y)=\left\langle\mathfrak{e}_{-X} \# f \# \mathfrak{e}_{X-Y}, h\right\rangle=\exp \left[\frac{i}{2} \sigma(X, Y)\right]\left\langle\Theta_{X}(f) \# \mathfrak{e}_{-Y}, h\right\rangle,
$$

which is different from the standard choice (the Short Time Fourier Transform)

$$
\left[\mathcal{V}_{h}(f)\right](X, Y)=\left\langle\mathfrak{e}_{-Y} \Theta_{X}(f), h\right\rangle .
$$

The main difference is the replacement of point-wise multiplication by the Weyl product, and this might be an advantage for studying the algebraic properties of the modulation mapping.

The next orthogonality relations justify formally Definition 2.1

Theorem 2.2. For $f, g, h, k \in L^{2}(\Xi)$, one has

$$
\left\langle\left\langle\overline{M_{h}^{B}(f)}, M_{k}^{B}(g)\right\rangle\right\rangle=\langle\bar{h}, k\rangle\langle\bar{f}, g\rangle .
$$

In particular, $\frac{1}{\|h\|} M_{h}^{B}$ is an isometry.

The proof will use several properties which are gathered in the next lemma. 
Lemma 2.3. (a) For any $f_{1}, f_{2}, f_{3} \in L^{2}(\Xi)$,

$$
\left\langle f_{1} \#^{B} f_{2}, f_{3}\right\rangle=\left\langle f_{1}, f_{2} \#^{B} f_{3}\right\rangle=\left\langle f_{2}, f_{3} \#^{B} f_{1}\right\rangle .
$$

The same is true if one of the three functions is replaced by $\mathfrak{e}_{X}$, for some $X \in \Xi$. (b) One has in the weak sense

$$
\int_{\Xi} d Z\left|\mathfrak{e}_{Z}\right\rangle\left\langle\mathfrak{e}_{-Z}\right|=1
$$

(c) For any $f, g \in L^{1}(\Xi)$,

$$
\int_{\Xi} \int_{\Xi} d Y d Z\left[\Theta_{Z}^{B}(f)\right](Y) g(Y)=\int_{\Xi} d Z f(Z) \int_{\Xi} d Y g(Y) .
$$

Proof. (a) We proved in 24 the case $f_{1}, f_{2}, f_{3} \in \mathcal{S}(\Xi)$. For the other cases one rewrites the proof more carefully or uses an approximation argument.

(b) One has for $f, g \in L^{2}(\Xi)$,

$$
\begin{aligned}
& \int_{\Xi} d Z\left\langle f, \mathfrak{e}_{Z}\right\rangle\left\langle\mathfrak{e}_{-Z}, g\right\rangle=(2 \pi)^{2 N} \int_{\Xi} d Z(\mathfrak{F} f)(Z)(\mathfrak{F} g)(-Z) \\
= & (2 \pi)^{2 N} \int_{\Xi} d Z(\mathfrak{F} f)(Z) \overline{(\mathfrak{F} \bar{g})(Z)}=\int_{\Xi} d Z f(Z) g(Z)=\langle f, g\rangle .
\end{aligned}
$$

(c) We apply Proposition 1.1 and use the Fourier inversion formula:

$$
\begin{gathered}
\int_{\Xi} \int_{\Xi} d Y d Z\left[\Theta_{Z}^{B}(f)\right](Y) g(Y)=\int_{\Xi} \int_{\Xi} d Y d Z\left[\left(\widetilde{\Omega}_{\mathcal{P}}^{B}[z]\right) \star \Theta_{Z}(f)\right](Y) g(Y) \\
=\int_{\Xi} \int_{\Xi} d Y d Z \int_{\mathcal{X}^{*}} d \xi\left(\widetilde{\Omega}_{\mathcal{P}}^{B}[z]\right)(y, \xi)\left[\Theta_{Z}(f)\right](y, \eta-\xi) g(Y) \\
=(2 \pi)^{-N} \int_{\mathcal{X}} d y \int_{\mathcal{X}} d z \int_{\mathcal{X}^{*}} d \eta \int_{\mathcal{X}^{*}} d \zeta \int_{\mathcal{X}} d x \int_{\mathcal{X}^{*}} d \xi e^{-i x \cdot \xi} \Omega^{B}[\mathcal{P}(y ; x, z)] \\
\cdot f(y-z, \eta-\xi-\zeta) g(y, \eta) \\
=(2 \pi)^{-N} \int_{\mathcal{X}} d y \int_{\mathcal{X}} d z \int_{\mathcal{X}^{*}} d \eta \int_{\mathcal{X}^{*}} d \zeta \int_{\mathcal{X}} d x \int_{\mathcal{X}^{*}} d \nu e^{i x \cdot \zeta} e^{i x \cdot(\nu-\eta)} \Omega^{B}[\mathcal{P}(y ; x, z)] \\
\quad \cdot f(y-z, \nu) g(y, \eta) \\
=\int_{\mathcal{X}} d y \int_{\mathcal{X}} d z \int_{\mathcal{X}^{*}} d \eta \int_{\mathcal{X}^{*}} d \nu \Omega^{B}[\mathcal{P}(y ; 0, z)] f(y-z, \nu) g(y, \eta) \\
=\int_{\Xi} d Z f(Z) \int_{\Xi} d Y g(Y) .
\end{gathered}
$$


Proof of Theorem 2.2. Using Lemma 2.3 we compute

$$
\begin{gathered}
\left\langle\left\langle\overline{M_{h}^{B}(f)}, M_{k}^{B}(g)\right\rangle\right\rangle=\int_{\Xi} \int_{\Xi} d X d Y\left\langle\overline{\mathfrak{e}_{-X} \#^{B} f \#^{B} \mathfrak{e}_{X-Y}}, \bar{h}\right\rangle\left\langle\mathfrak{e}_{-X} \#^{B} g \#^{B} \mathfrak{e}_{X-Y}, k\right\rangle \\
=\int_{\Xi} \int_{\Xi} d X d Y\left\langle\mathfrak{e}_{Y-X} \#^{B} \bar{f} \#^{B} \mathfrak{e}_{X}, \bar{h}\right\rangle\left\langle\mathfrak{e}_{-X} \#^{B} g \#^{B} \mathfrak{e}_{X-Y}, k\right\rangle \\
=\int_{\Xi} \int_{\Xi} d X d Y\left\langle\bar{f} \#^{B} \mathfrak{e}_{X} \#^{B} \bar{h}, \mathfrak{e}_{Y-X}\right\rangle\left\langle\mathfrak{e}_{X-Y}, k \#^{B} \mathfrak{e}_{-X} \#^{B} g\right\rangle \\
=\int_{\Xi} d X\left\langle\bar{f} \#^{B} \mathfrak{e}_{X} \#^{B} \bar{h}, k \#^{B} \mathfrak{e}_{-X} \#^{B} g\right\rangle=\int_{\Xi} d X\left\langle\mathfrak{e}_{X} \#^{B} \bar{h} \#^{B} k, \mathfrak{e}_{-X} \#^{B} g \#^{B} \bar{f}\right\rangle \\
=\int_{\Xi} d X\left\langle\Theta_{X}^{B}\left(\bar{h} \#^{B} k\right), g \#^{B} \bar{f}\right\rangle=\int_{\Xi} d X\left(\bar{h} \#^{B} k\right)(X) \int_{\Xi} d Y\left(g \#^{B} \bar{f}\right)(Y)=\langle\bar{h}, k\rangle\langle\bar{f}, g\rangle .
\end{gathered}
$$

Corollary 2.4. We have the inversion formula

$$
\left(M_{k}^{B}\right)^{*} M_{h}^{B}=\langle h, \bar{k}\rangle \mathrm{id} .
$$

The adjoint $\left(M_{h}^{B}\right)^{*}: L^{2}(\Xi \times \Xi) \rightarrow L^{2}(\Xi)$ is given explicitly by

$$
\begin{gathered}
\left(M_{h}^{B}\right)^{*}(G):=\int_{\Xi} \int_{\Xi} d X d Y G(X, Y) \mathfrak{e}_{X} \#^{B} h \#^{B} \mathfrak{e}_{Y-X} \\
=\int_{\Xi} \int_{\Xi} d X d Y G(X, Y)\left(\Theta_{X, Y}^{B}\right)^{-1}(h) .
\end{gathered}
$$

Remark. Theorem 2.2 suggests defining

$$
M^{B}: L^{2}(\Xi \times \Xi) \cong L^{2}(\Xi) \otimes L^{2}(\Xi) \rightarrow L^{2}(\Xi \times \Xi), \quad M^{B}(f \otimes h):=M_{h}^{B}(f) .
$$

One has $M_{h}^{B}=M^{B} \circ J_{h}$, where for any $h \in L^{2}(\Xi)$ we set

$$
J_{h}: L^{2}(\Xi) \rightarrow L^{2}(\Xi \times \Xi), \quad J_{h}(f):=f \otimes h .
$$

The adjoint is given by

$$
J_{h}^{*}: L^{2}(\Xi \times \Xi) \rightarrow L^{2}(\Xi), \quad\left[J_{h}^{*}(F)\right](X):=\langle F(X, \cdot), h(\cdot)\rangle,
$$

and it satisfies

$$
J_{k}^{*} J_{h}=\langle h, k\rangle \mathrm{id}, \quad J_{h} J_{k}^{*}=1 \otimes(|h\rangle\langle k|)=1 \otimes \operatorname{Int}_{h \otimes k} .
$$

While $M^{B}$ is an isomorphism, $\|h\|^{-1} J_{h}$ is an isometry with range $L^{2}(\Xi) \otimes\{h\}$.

We turn now to the algebraic properties of the magnetic modulation mapping. On functions : $\Xi \times \Xi \rightarrow \mathbb{C}$ we use the crossed product composition

$$
(F \diamond G)(X, Y):=\int_{\Xi} d Z F(X, Z) G(X-Z, Y-Z)
$$

and the involution $F^{*}(X, Y):=\overline{F(X-Y,-Y)}$. The "crossed product" feature can be seen if we write (2.4) as

$$
(F \diamond G)(Y):=\int_{\Xi} d Z F(Z) \Theta_{Z}[G(Y-Z)] .
$$

This is an equality between functions defined on $\Xi$, so it must be evaluated on $X \in \Xi$ by using notation such as $[F(Z)](X):=F(X, Z)$. The action of $\Xi$ on itself given by $\Theta_{Z}(X):=X+Z$ is transferred to functions by $\Theta_{Z}(g):=g \circ \Theta_{-Z}$. For various function spaces on $\Xi$ one can define $C^{*}$-dynamical systems, and they 
generate naturally crossed product $C^{*}$-algebras of functions or distributions defined on $\Xi \times \Xi$. We refer to 33 . for general information on this topic; we are going to study the connection of crossed products with magnetic modulation spaces in a further publication.

For the moment we only notice that $L^{2}(\Xi \times \Xi)$ is a ${ }^{*}$-algebra with the structure indicated above. To see this, one might recall the kernel multiplication

$$
(K \tilde{\diamond} L)(X, Y):=\int_{\Xi} d Z K(X, Z) L(Z, Y)
$$

and the involution $K^{\tilde{*}}(X, Y):=\overline{K(Y, X)}$ and perform the change of variables $(X, Y) \mapsto(X, X-Y)$.

Theorem 2.5. If $h \#^{B} h=h=\bar{h} \neq 0$, then $M_{h}^{B}: L^{2}(\Xi) \rightarrow L^{2}(\Xi \times \Xi)$ is an injective morphism of ${ }^{*}$-algebras.

Proof. We use (2.1) and (2.2) to show that

$$
M_{h}^{B}(f) \diamond M_{k}^{B}(g)=M_{k \#^{B} h}^{B}\left(f \#^{B} g\right),
$$

and then take $h=k$. One has

$$
\begin{aligned}
& {\left[M_{h}^{B}(f) \diamond M_{k}^{B}(g)\right](X, Y)=\int_{\Xi} d Z\left[M_{h}^{B}(f)\right](X, Z)\left[M_{k}^{B}(g)\right](X-Z, Y-Z)} \\
& \quad=\int_{\Xi} d Z\left\langle\mathfrak{e}_{-X} \#^{B} f \#^{B} \mathfrak{e}_{X-Z}, h\right\rangle\left\langle\mathfrak{e}_{Z-X} \#^{B} g \#^{B} \mathfrak{e}_{X-Y}, k\right\rangle \\
& \quad=\int_{\Xi} d Z\left\langle h \#^{B} \mathfrak{e}_{-X} \#^{B} f, \mathfrak{e}_{X-Z}\right\rangle\left\langle\mathfrak{e}_{Z-X}, g \#^{B} \mathfrak{e}_{X-Y} \#^{B} k\right\rangle \\
& =\left\langle h \#^{B} \mathfrak{e}_{-X} \#^{B} f, g \#^{B} \mathfrak{e}_{X-Y} \#^{B} k\right\rangle \\
& =\left\langle\mathfrak{e}_{-X} \#^{B}\left(f \#^{B} g\right) \#^{B} \mathfrak{e}_{X-Y}, k \#^{B} h\right\rangle=\left[M_{k \#^{B} h}^{B}\left(f \#^{B} g\right)\right](X, Y) .
\end{aligned}
$$

For the involution:

$$
\begin{gathered}
{\left[M_{h^{*}}^{B}\left(f^{*}\right)\right](X, Y)=\left\langle\mathfrak{e}_{-X} \#^{B} \bar{f} \#^{B} \mathfrak{e}_{X-Y}, \bar{h}\right\rangle} \\
=\overline{\left\langle\mathfrak{e}_{Y-X} \#^{B} f \#^{B} \mathfrak{e}_{X}, h\right\rangle}=\overline{\left[M_{h}^{B}(f)\right](X-Y,-Y)}=\left[M_{h}^{B}(f)\right]^{*}(X, Y) .
\end{gathered}
$$

The injectivity follows from Theorem 2.2

Remark. One could also use the composition law

$\square^{B}: L^{2}(\Xi \times \Xi) \times L^{2}(\Xi \times \Xi) \rightarrow L^{2}(\Xi \times \Xi), \quad(f \otimes h) \square^{B}(g \otimes k):=\left(f \#^{B} g\right) \otimes\left(k \#^{B} h\right)$

and the usual involution on $L^{2}(\Xi \times \Xi)$ given by complex conjugation. Then

$$
M^{B}:\left(L^{2}(\Xi \times \Xi), \square^{B}\right) \rightarrow\left(L^{2}(\Xi \times \Xi), \diamond\right)
$$

is an isomorphism of ${ }^{*}$-algebras.

\section{Connections with the Bargmann transform}

The magnetic analog of the Bargmann transform requires a suitable family of coherent states. The main idea for introducing them will be to use the magnetic Weyl system (1.7) to propagate a given state, corresponding to $X=0$, to a family of states indexed by the points $X$ of phase space. In order to insure gauge-covariance, this state must have a good a priori dependence of the vector potential $A$. Of course this seems to fit the group-theoretical strategy to generate coherent states, but we 
stress that (1.8) reduces to the definition of a projective representation only in the very simple case of a constant magnetic field.

We note that with a proper implementation of Planck's constant $\hbar$, one proves ([25]) convergence of the quantum algebra of observables to the classical one in the sense of strict deformation quantization (cf. 29, 22, ). We intend to study in a future publication the dependence of coherent states and the associated Berezin-Toeplitz operators on the Planck constant, in the framework of deformation quantization (cf. [20, 21, 22]).

Let us fix a unit vector, $v \in \mathcal{H}:=L^{2}(\mathcal{X})$. For any choice of a continuous vector potential $A$ generating the magnetic field $B$ and for any $Y \in \Xi$, we define the family of magnetic coherent vectors

$$
v^{A} \equiv v^{A}(0):=e^{i \Gamma^{A}([0, Q])} v, \quad v^{A}(Y):=\mathfrak{o p}^{A}(-Y) v^{A} .
$$

Explicitly

$$
\left[v^{A}(Y)\right](x)=e^{i\left(x-\frac{y}{2}\right) \cdot \eta} e^{-i \Gamma^{A}([x, x-y])} e^{i \Gamma^{A}([0, x-y])} v(x-y) .
$$

Note that for the standard Gaussian $v(x)=\pi^{-N / 4} e^{-x^{2} / 2}$ and for $A=0$, one gets the usual coherent states of Quantum Mechanics (see [1]). It is easy to show that in the weak sense

$$
\int_{\Xi} \frac{d Y}{(2 \pi)^{N}}\left|v^{A}(Y)\right\rangle\left\langle v^{A}(Y)\right|=1
$$

We are not going to prove this simple result, since it will not be needed in the sequel.

Since the pure state space of $\mathbb{K}(\mathcal{H})$ can be identified with $\mathbb{P}(\mathcal{H})$ (the family of all self-adjoint one-dimensional projections in $\mathcal{H}$ ) and considering the isomorphism $\mathfrak{O p}^{A}: \mathfrak{A}^{B}(\Xi) \rightarrow \mathbb{K}(\mathcal{H})$, we introduce families of coherent states on the two $C^{*}$ algebras:

Definition 3.1. For any $Z \in \Xi$ we define

$$
\mathfrak{v}^{A}(Z): \mathbb{K}(\mathcal{H}) \rightarrow \mathbb{C}, \quad \mathfrak{v}^{B}(Z): \mathfrak{A}^{B}(\Xi) \rightarrow \mathbb{C}
$$

by

$$
\left[\mathfrak{v}^{A}(Z)\right](S):=\operatorname{Tr}\left(\left|v^{A}(Z)\right\rangle\left\langle v^{A}(Z)\right| S\right)=\left\langle v^{A}(Z), S v^{A}(Z)\right\rangle, \quad \forall S \in \mathbb{K}(\mathcal{H})
$$

and

$$
\left[\mathfrak{v}^{B}(Z)\right](f):=\left[\mathfrak{v}^{A}(Z)\right]\left[\mathfrak{O p}^{A}(f)\right]=\left\langle v^{A}(Z), \mathfrak{O p}^{A}(f) v^{A}(Z)\right\rangle, \quad \forall f \in \mathfrak{A}^{B}(\Xi) .
$$

One can write

$$
\begin{aligned}
{\left[\mathfrak{v}^{B}(Z)\right](f) } & =\left\langle v^{A}, \mathfrak{o p}^{A}(Z) \mathfrak{O p}^{A}(f) \mathfrak{o p} \mathfrak{p}^{A}(-Z) v^{A}\right\rangle \\
& =\left\langle v^{A}, \mathfrak{O p} \mathfrak{p}^{A}\left(\mathfrak{e}_{Z} \#^{B} f \#^{B} \mathfrak{e}_{-Z}\right) v^{A}\right\rangle .
\end{aligned}
$$

Using (1.9) and setting $\mathfrak{v}^{B}:=\mathfrak{v}^{B}(0)$, one has $\mathfrak{v}^{B}(Z)=\mathfrak{v}^{B} \circ \Theta_{Z}^{B}$. The intrinsic notation $\mathfrak{v}^{B}(Z)$ is justified by a straightforward computation based on Stokes' 
Theorem, leading to

$$
\begin{gathered}
{\left[\mathfrak{v}^{B}(Z)\right](f)} \\
=(2 \pi)^{-N} \int_{\mathcal{X}} \int_{\mathcal{X}} \int_{\mathcal{X}^{*}} d x d y d \xi e^{i(x-y) \cdot(\xi-\zeta)} f\left(\frac{x+y}{2}, \xi\right) \overline{v(x-z)} v(y-z) \\
\cdot \exp \left\{i\left[\Gamma^{B}(\langle y, x, x-z\rangle)+\Gamma^{B}(\langle y, x-z, 0\rangle)+\Gamma^{B}(\langle y, 0, y-z\rangle)\right]\right\} .
\end{gathered}
$$

A convenient setting is obtained after making a unitary transformation, generalizing the classical Bargmann transformation; the associated Bargmann-type space is a Hilbert space with reproducing kernel.

Definition 3.2. (a) The mapping $\mathcal{U}_{v}^{A}: L^{2}(\mathcal{X}) \rightarrow L^{2}\left(\Xi ; \frac{d X}{(2 \pi)^{N}}\right)$,

$$
\left(\mathcal{U}_{v}^{A} u\right)(X):=\left\langle v^{A}(X), u\right\rangle=\left\langle v^{A}, \mathfrak{o p}^{A}(X) u\right\rangle
$$

is called the Bargmann transformation corresponding to the family of coherent states $\left(v^{A}(X)\right)_{X \in \Xi}$.

(b) The subspace $\mathcal{K}_{v}^{A}:=\mathcal{U}_{v}^{A} L^{2}(\mathcal{X}) \subset L^{2}(\Xi)$ is called the Bargmann space corresponding to the family of coherent states $\left(v^{A}(X)\right)_{X \in \Xi}$.

The proofs of the statements below are straightforward and not specific to our magnetic framework (see 22, section II.1.5, for instance):

Proposition 3.3. (a) $\mathcal{U}_{v}^{A}$ is an isometry with adjoint

$$
\left(\mathcal{U}_{v}^{A}\right)^{*}: L^{2}(\Xi) \rightarrow L^{2}(\mathcal{X}), \quad\left(\mathcal{U}_{v}^{A}\right)^{*} \Phi:=\int_{\Xi} \frac{d X}{(2 \pi)^{N}} \Phi(X) v^{A}(X)
$$

and final projection $P_{v}^{A}:=\mathcal{U}_{v}^{A}\left(\mathcal{U}_{v}^{A}\right)^{*} \in \mathbb{P}\left[L^{2}(\Xi)\right]$, with $P_{v}^{A} L^{2}(\Xi)=\mathcal{K}_{v}^{A}$.

(b) The kernel of this projection

$$
K_{v}^{A}: \Xi \times \Xi \rightarrow \mathbb{C}, \quad K_{v}^{A}(X, Y):=\left\langle v^{A}(X), v^{A}(Y)\right\rangle
$$

is a continuous function and it is a reproducing kernel for $\mathcal{K}_{v}^{A}$ :

$$
\Phi(X)=\int_{\Xi} \frac{d X}{(2 \pi)^{N}} K_{v}^{A}(X, Y) \Phi(Y), \quad \forall X \in \Xi, \quad \forall \Phi \in \mathcal{K}_{v}^{A} .
$$

(c) The Bargmann space is composed of continuous functions and contains all the vectors $K_{v}^{A}(X, \cdot), X \in \Xi$. The evaluation maps $\mathcal{K}_{v}^{A} \ni \Phi \rightarrow \Phi(X) \in \mathbb{C}$ are all continuous.

For various types of vectors $u, v: \Xi \rightarrow \mathbb{C}$ we define the magnetic Wigner transform $V_{u, v}^{A}$ by

$$
\left\langle u, \mathfrak{O p}^{A}(f) v\right\rangle=: \int_{\Xi} d X f(X) V_{u, v}^{A}(X) .
$$

One easily gets $V_{v, u}^{A}=(2 \pi)^{N / 2}[(1 \otimes \mathcal{F}) \circ C]\left[\bar{\gamma}^{A} \cdot(u \otimes \bar{v})\right]$, with $\gamma^{A}(a, b):=e^{-i \Gamma^{A}([a, b])}$, which may also be written

$$
V_{v, u}^{A}(z, \zeta)=\int_{\mathcal{X}} d y e^{i y \cdot \zeta} \bar{\gamma}^{A}\left(z+\frac{y}{2}, z-\frac{y}{2}\right) u\left(z+\frac{y}{2}\right) \bar{v}\left(z-\frac{y}{2}\right) .
$$

It follows that $u, v \in L^{2}(\mathcal{X}) \Longrightarrow V_{u, v}^{A} \in L^{2}(\Xi)$. By a direct computation one gets $|u\rangle\langle v|=\mathfrak{O p}^{A}\left(V_{u, v}^{A}\right)$, which describes all the rank-one operators in $\mathcal{H}$. This 
suggests studying the modulation mapping $M_{h}^{B}$ in the particular case in which $h$ is the Wigner transform $h(B, v):=V_{v^{A}, v^{A}}^{A}$, given explicitly by

$$
[h(B, v)](z, \zeta)=\int_{\mathcal{X}} d y e^{i y \cdot \zeta} \exp \left[i \Gamma^{B}\left(\left\langle 0, z+\frac{y}{2}, z-\frac{y}{2}\right\rangle\right)\right] v\left(z+\frac{y}{2}\right) \overline{v\left(z-\frac{y}{2}\right)} .
$$

Let us also set $\mathbb{U}_{v}^{A}[T]:=\mathcal{U}_{v}^{A} T\left(\mathcal{U}_{v}^{A}\right)^{*}$. We denote by $R E P$ the representation of $L^{2}(\Xi \times \Xi)$ in $L^{2}(\Xi)$ given by

$$
[R E P(F) \Phi](X):=\int_{\Xi} d Y F(X, X-Y) \Phi(Y) .
$$

Proposition 3.4. One has

$$
R E P \circ M_{h(B, v)}^{B}=\mathbb{U}_{v}^{A} \circ \mathfrak{O p}^{A} .
$$

Proof. For any $f \in L^{2}(\Xi)$ we have

$$
\begin{aligned}
& {\left[\mathcal{U}_{v}^{A} \mathfrak{O p}^{A}(f)\left(\mathcal{U}_{v}^{A}\right)^{*} \Phi\right](X)=\left\langle v^{A}(X), \mathfrak{O p}^{A}(f)\left(\mathcal{U}_{v}^{A}\right)^{*} \Phi\right\rangle} \\
& =\left\langle v^{A}(X), \mathfrak{O} \mathfrak{p}^{A}(f) \int_{\Xi} \frac{d Y}{(2 \pi)^{N}} \Phi(Y) v^{A}(Y)\right\rangle \\
& =\int_{\Xi} \frac{d Y}{(2 \pi)^{N}} \Phi(Y)\left\langle v^{A}(X), \mathfrak{O p} \mathfrak{p}^{A}(f) v^{A}(Y)\right\rangle \\
& =\int_{\Xi} \frac{d Y}{(2 \pi)^{N}} \Phi(Y)\left\langle v^{A}, \mathfrak{O p}^{A}\left(\mathfrak{e}_{-X} \#^{B} f \#^{B} \mathfrak{e}_{Y}\right) v^{A}\right\rangle \\
& =\int_{\Xi} \frac{d Y}{(2 \pi)^{N}}\left[M_{h(B, v)}^{B}(f)\right](X, X-Y) \Phi(Y) \\
& =\left(\left[\left(R E P \circ M_{h(B, v)}^{B}\right)(f)\right](\Phi)\right)(X) .
\end{aligned}
$$

Remark. We can further compose $M_{h(B, v)}^{B}$ with a partial Fourier transformation $1 \otimes \mathfrak{F}: L^{2}(\Xi \times \Xi) \rightarrow L^{2}(\Xi \times \Xi)$. Since $O P:=R E P \circ(1 \otimes \mathfrak{F})$ is essentially the Kohn-Nirenberg pseudodifferential calculus in $\mathbb{R}^{2 N}$, one gets a nice interpretation for the operator $\mathfrak{O p}^{A}(f)$ : its magnetic Bargmann transform associated to $(A, v)$ is a usual pseudodifferential operator with symbol $(1 \otimes \mathfrak{F}) \circ M_{h(B, v)}^{B}$. This can also be considered a nice interpretation of the magnetic modulation mapping.

\section{ACKNOWLEDGEMENTS}

The first author would like to thank the members of the NuHAG group and especially Professor Hans Feichtinger for hospitality and many useful discussions.

\section{REFERENCES}

1. S.T. Ali, J-P. Antoine, J-P. Gazeau and U. A. Müller, Coherent States and Their Generalizations: A Mathematical Overview, Rev. Math. Phys. 7 (1990), 1013-1104. MR.1359988 (97b:81040)

2. D. Beltită and I. Beltită, Magnetic Pseudodifferential Weyl Calculus on Nilpotent Lie Groups, Ann. Global Anal. Geom. 36 (3) (2009), 293-322. MR2544305

3. D. Beltită and I. Beltită, Uncertainty Principles for Magnetic Structures on Certain Coadjoint Orbits, J. Geom. Phys. 60 (1) (2010), 81-95.

4. A. Boulkhemair, Remarks on a Wiener Type Pseudodifferential Algebra and Fourier Integral Operators, Math. Res. Lett. 4 (1) (1997), 53-67. MR.1432810 (98f:47057)

5. H. G. Feichtinger, On a New Segal Algebra, Monatsh. Mat. 92 (4) (1981), 269-289. MR643206 (83a:43002) 
6. H. G. Feichtinger, Modulation Spaces on Locally Compact Abelian Groups, in Proceedings of "International Conference on Wavelets and Applications 2002", 99-140, Chenai, India. Updated version of a technical report, University of Vienna, 1983.

7. G. Fendler, K. Gröchenig and M. Leinert, Convolution-Dominated Operators on Discrete Groups, Int. Eq. Op. Th. 61 (2008), 493-509. MR2434338 (2009m:47085)

8. G. B. Folland, Harmonic Analysis in Phase Space, Princeton Univ. Press, Princeton, NJ, 1989. MR $983366(92 \mathrm{k}: 22017)$

9. K. Gröchenig, Foundations of Time-Frequency Analysis, Birkhäuser Boston Inc., Boston, MA, 2001. MR1843717 (2002h:42001)

10. K. Gröchenig, Time-Frequency Analysis of Sjöstrand Class, Revista Mat. Iberoam. 22 (2) (2006), 703-724. MR2294795 (2008b:35308)

11. K. Gröchenig, Composition and Spectral Invariance of Pseudodifferential Operators on Modulation Spaces, J. Anal. Math. 98 (2006), 65-82. MR2254480 (2007f:47044)

12. K. Gröchenig, A Pedestrian Approach to Pseudodifferential Operators, in C. Heil, editor, Harmonic Analysis and Applications, Birkhäuser, Boston, 2006. In honour of John J. Benedetto. MR.2249309 (2007i:35257)

13. K. Gröchenig and C. Heil, Modulation Spaces and Pseudodifferential Operators, Int. Eq. Op. Th. 34 (1999), 439-457. MR1702232(2001a:47051)

14. K. Gröchenig and J. Toft, Localization Operator Representation of Modulation Spaces, preprint.

15. B. C. Hall, Quantum Mechanics in Phase Space, Contemporary Mathematics, 214, Amer. Math. Soc., Providence, RI, 1997, 47-62. MR1601217 (99e:22015)

16. C. Heil, J. Ramanathan, and P. Topiwala, Singular Values of Compact Pseudodifferential Operators, J. Funct. Anal. 150 (2) (1997), 426-452. MR1479546 (98k:47102)

17. V. Iftimie, M. Măntoiu and R. Purice, Magnetic Pseudodifferential Operators, Publ. RIMS 43 (2007), 585-623. MR2361789 (2009h:35469)

18. V. Iftimie, M. Măntoiu and R. Purice, A Beals-Type Criterion for Magnetic Pseudodifferential Operators, Commun. PDE, to appear.

19. M. V. Karasev and T. A. Osborn, Symplectic Areas, Quantization and Dynamics in Electromagnetic Fields, J. Math. Phys. 43 (2002), 756-788. MR1878969(2002m:81120)

20. N. P. Landsman, Classical Behaviour in Quantum Mechanics: A Transition Probability Approach, Int. J. of Mod. Phys. B (1996), 1545-1554. MR.1405182 (97g:81003)

21. N. P. Landsman, Poisson Spaces with a Transition Probability, Rev. Math. Phys. 9 (1997), 29-57. MR 1426545 (98b:81014)

22. N. P. Landsman, Mathematical Topics between Classical and Quantum Mechanics, SpringerVerlag, New York, 1998. MR 1662141 (2000g:81081)

23. M. Lein, M. Măntoiu and S. Richard, Magnetic Pseudodifferential Operators with Coefficients in $C^{*}$-algebras, preprint arXiv:0901.3704, to appear in Proc. of the RIMS.

24. M. Măntoiu and R. Purice, The Magnetic Weyl Calculus, J. Math. Phys. 45 (4) (2004), 1394-1417. MR2043834(2005j:81085)

25. M. Măntoiu and R. Purice, Strict Deformation Quantization for a Particle in a Magnetic Field, J. Math. Phys. 46 (5) (2005). MR2142981 (2006b:81139)

26. M. Măntoiu and R. Purice, The Mathematical Formalism of a Particle in a Magnetic Field, Proceedings of the Conference QMath. 9, Giens, France, Lecture Notes in Math., vol. 690, Springer-Berlin, 2006. MR2235706 (2007c:81098)

27. M. Măntoiu, R. Purice and S. Richard, Twisted Crossed Products and Magnetic Pseudodifferential Operators, Proceedings of the Conference of Sinaia (Romania), Theta Foundation, 2005. MR2238287 (2008d:46096)

28. M. Măntoiu, R. Purice and S. Richard, Spectral and Propagation Results for Magnetic Schrödinger Operators; a $C^{*}$-Algebraic Approach, J. Funct. Anal. 250 (2007), 42-67. MR.2345905 (2009a:46128)

29. M. A. Rieffel, Deformation Quantization for Actions of $\mathbb{R}^{d}$, Memoirs Amer. Math. Soc. 106 (1993), no. 506. MR 1184061 (94d:46072)

30. J. Sjöstrand, An Algebra of Pseudodifferential Operators, Math. Res. Lett. 1 (2) (1994), 185-192. MR.1266757 (95b:47065)

31. J. Sjöstrand, Wiener Type Algebras of Pseudodifferential Operators, in Séminaire sur les Équations aux Dérivées Partielles, 1994-1995, Exp. No. IV, 21, École Polytech., Palaiseau, 1995. MR $1362552(96 \mathrm{j}: 47049)$ 
32. J. Toft, Subalgebras to a Wiener Type Algebra of Pseudo-differential Operators, Ann. Inst. Fourier (Grenoble) 51 (5) (2001), 1347-1383. MR.1860668 (2002h:47071)

33. D. Williams, Crossed Products of $C^{*}$-Algebras, Math. Surveys and Monographs, 134, American Mathematical Society, Providence, RI, 2007. MR2288954 (2007m:46003)

Departamento de Matematicas, Universidad de Chile, las Palmeras 3425, Casilla 653, Santiago, Chile

E-mail address: Marius.Mantoiu@imar.ro

E-mail address: mantoiu@uchile.cl

Institute of Mathematics Simion Stollow of the Romanian Academy, P.O. Box 1-764, Bucharest, RO-70700, Romania

E-mail address: Radu.Purice@imar.ro 